\title{
BENIFICIAL EFFECT OF STACHYDRINE ON THE TRAUMATIC BRAIN INJURY INDUCED NEURODEGENERATION BY ATTENUATING THE EXPRESSIONS OF AKT/MTOR/ PI3K AND TLR4/NFK-B PATHWAY
}

Abstract

Present investigation aims to explore the protective effect of stachydrine against traumatic brain injury (TBI) and also investigate the molecular mechanism of its action. TBI was induced by the fall a hammer $(450 \mathrm{~g})$ from the height of $1.5 \mathrm{~m}$. and later stachydrine was administered for 2 weeks starting $2 \mathrm{hr}$ after the induction of TBI. Effect of stachydrine was determined by estimating modified neurological severity score (mNSS), percentage of water content in the brain and cognitive dysfunction in TBI rats. Moreover western blot assay, histopathology and enzyme linked immunosorbent assay (ELISA) tests were used to determine the effect of stachydrine on TBI injured rats. Result of the report suggests that stachydrine reduces the mNSS and percentage of water content in the brain and also attenuates the cognitive dysfunction in TBI injured rats. However data of western blot assay reports that stachydrine reduces the expression of $\mathrm{PI} 3 \mathrm{~K} / \mathrm{m}$-TOR/Akt pathway in the brain tissues of TBI rats. Concentration of interleukin (IL-1 $\beta$ ), tumor necrosis factor- $\alpha$ (TNF- $\alpha$ ) and interferon gamma (INF- $\gamma$ ) was reduces in stachydrine treated group than TBI group. Moreover expression of Nuclear factor- $\mathrm{KB} /$ Toll-like receptor 4 (NF$\mathrm{KB} / \mathrm{TLR}-4)$ protein was also decreased in stachydrine treated group than TBI group. Histopathology study on brain tissue reveals that the percentage of apoptotic cells was also reduced in stachydrine treated group than TBI group. Data of this investigation concludes that stachydrine protects the neuronal injury by attenuating the phosphatidylinositide 3-kinases/mammalian target of rapamycin/Protein kinase B (PI3K/m-TOR/Akt) and NF-KB/ TLR-4 pathway in TBI injured rats.

Keywords Stachydrine $\cdot$ Traumatic brain injury • Apoptosis • Inflammation • TLR-4

\section{Introduction}

In the modern society traumatic brain injury (TBI) is a major health problem that causes disability and death in the young aged population [1]. Center for Disease Control and Prevention reported that the patient suffering from TBI enhancing every year as in USA approximately 1.7 million patients reported with it [2]. Pathogenesis of TBI suggest that injury to brain immediately due to trauma called as primary injury, while secondary injury occurs due to alteration in molecular and cellular events in neuronal cells [3]. Several events such as enhancement in the permeability of vessels, oxidative stress, inflammation and mitochondrial dysfunction were observed in the TBI which leads to cell death and alteration in cognitive function [4]. Literature reported that inflammatory pathway such as NF- $\mathrm{KB} / \mathrm{TLR}-4$ signaling pathway and PI3K/Akt apoptotic pathway of autophagy plays an important role in the management of TBI [5].

Alternative medicine in the recent past years has shown potential role in the treatment of chronic disease. Stachydrine is chemically an alkaloid isolated from Leonurus heterophyllus (Labiatae) [6]. Leonurus heterophyllus traditionally used for the several medicinal purpose such as antiplatelet, improves the heart function and coronary flow in China [7]. Moreover Stachydrine is reported for several pharmacological activities such as anti inflammatory, anti cancer, cardioprotective and cerebral ischemia [8-10]. It was reported that stachydrine has a strong effect on inflammatory pathway [11]. Thus present study determines the protective effect of stachydrine on neuronal injury.
Nianzu Yu,

$\mathrm{Si} \mathrm{Hu}$,

Zheng Hao*

Department of Neurosurgery

The First Affiliated Hospital of Nanchang University, Nanchang 330006, China
Received 23 September 2018 accepted 14 October 2018

\section{Material and methods}

\section{Animals}

Albino rat (Age: 3 weeks; Weight: 17-23g) was used in the given study. All the animals were procured from Taizhou University Medical School, China. Standard guidelines (Humidity: $60 \pm 5 \%$; Temperature: $24 \pm 3^{\circ} \mathrm{C}$ ) were used to store the animals for $12 \mathrm{hr}$ light and dark cycle. Protocols of the investigation were approved by ethics committee of The First Affiliated Hospital of Nanchang University, China (IACUC/ FAHNU/2017/08) and the given study followed the guidelines of Association for the Assessment and Accreditation of Laboratory Animal Care International (AAALAC) for experimentation and animal use.

\section{Chemicals}

Stachydrine was purchased from MedChem 
Express, USA and isoflurane was procured from Abbott Laboratories, USA. t-PI3K, p-PI3K, t-Akt, p-Akt, p-mTOR, t-mTOR, Bcl-2, Bax, LC-3, TLR-4, $\mathrm{NF}-\kappa \mathrm{B}$ and $\beta$-actin was procured from Abcam, California, USA. ELISA kits were purchased from Ebioscience Company, USA.

\section{Experimental}

Animals were anesthetized before the surgery by administering the pentobarbital $60 \mathrm{mg} /$ kg i.p. and skull of animals were exposed by making an incision to medline. Later a disc of steel having the dimension $3 \mathrm{~mm}$ thickness and $10 \mathrm{~mm}$ diameter placed at biparietal and coronal suture. Rats were placed on the cushion and a copper hammer of $450 \mathrm{~g}$ was fall from the height of $1.5 \mathrm{~m}$. Thereafter skull was cleaned properly and sutured the same.

All the animals were divided in to five different groups such as Normal group which is shame operated group receives only saline solution; TBI group which receives saline solution; stachydrine 30 and $60 \mathrm{mg} / \mathrm{kg}$ group which receives stachydrine 30 and $60 \mathrm{mg} / \mathrm{kg}$, i.p. $2 \mathrm{hr}$ after the TBI for the duration of one week.

\section{Estimation of neurological function} Modified Neurological Severity Score (mNSS) was used to determine balance, touch, vision, abnormal behavior, muscle mass, sensation and motion. mNSS was calculated on the 0-18 scale, as 0 shows the normal brain function and 18 shows the sever defect in the neurological function. Neurological functions were determined from day 1, 3, 7 and 14 .

Determination of water content in the brain

All the animals were sacrificed and the brain was isolated from the rats and analytical balance was used to determine the wet weight of brain and later dry weight of brain tissue was observed by drying it in oven at $100^{\circ} \mathrm{C}$ for one day.

$\%$ water content brain $=$ (wet weight - dry weight)/wet weight $\times 100 \%$.

\section{Determination of behavioral changes} Morris water maze apparatus was used to determine the behavioral changes. Water maze having height and diameter of 0.5 and $1.2 \mathrm{~m}$ respectively and the platform was depth at
$15.5 \mathrm{~cm}$. Four quadrants were created in the apparatus with thread and in the one of the quadrant platform was placed in such a way that it was not able to visualized. Observation of swimming behavior was done for the duration of six days continuously and escape latency was determined. Later by escaping the platform spatial memory of each rat was determined and effect of stachydrine was also estimated in the TBI injured rats.

\section{Nissl staining}

Isolated brain tissues were fixed by using $4 \%$ formalin and further tissues were embedded liquid paraffin. Microtome was used to section the brain tissues of $4 \mu \mathrm{m}$ thickness and xylene was used to de-paraffinized the tissue. These tissue sections were treated with alcohol to rehydrate the tissues. Nissl staining was used to stain the brain tissues for $5 \mathrm{~min}$. Section of tissues were observed by using trinocular

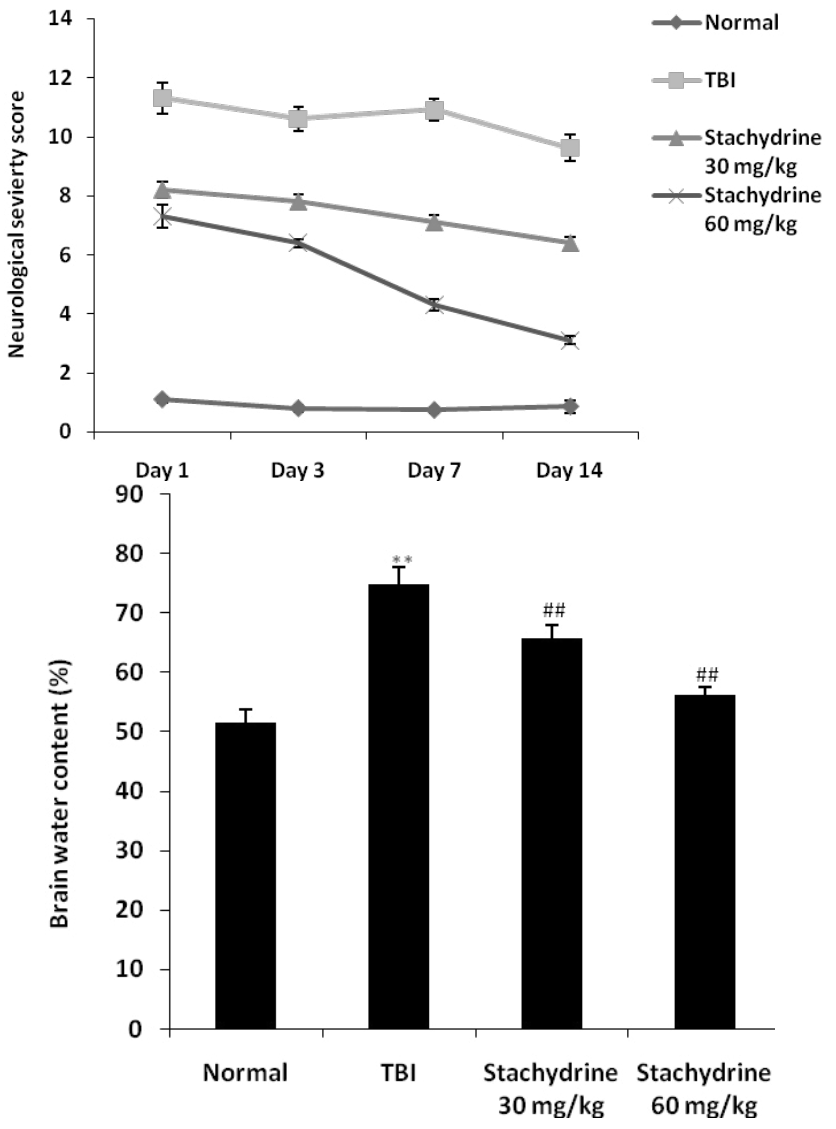

Fig.1. Stachydrine effect on the mNSS and percentage of water content in the brain of TBI injured rats Mean \pm $\operatorname{SEM}(\mathrm{n}=10)$, ${ }^{* *} \mathrm{p}<0.01$ than normal group; ${ }^{\# \#} \mathrm{p}<0.01$ than TBI group microscope and evaluated randomly by pathologist.

\section{Western blot assay}

Brain tissues of injured location were separated out and tissue lysis was done using lysis buffer. Supernatant from the lysed tissue was separated out by centrifuging the lysate for the period of $5 \mathrm{~min}$ at 10000 RPM. Later sodium dodecyl sulfate-polyacrylamide gel electrophoresis was used to separate the total protein and then separated protein is filtered on nitrocellulose membrane. Further membrane was incubated with $I^{\text {ry }}$ antibodies like t-PI3K (Dilution at a ratio of 1:500), $\mathrm{p}$-PI3K (Dilution at a ratio of 1:500), t-Akt (Dilution at a ratio of 1:500), p-Akt (Dilution at a ratio of 1:500), p-mTOR (Dilution at a ratio of 1:500), t-mTOR (Dilution at a ratio of 1:500), LC-3 (Dilution at a ratio of 1:500), TLR4 (Dilution at a ratio of 1:1000), NF-kB (Dilution at a ratio of $1: 1500$ ) and $\beta$-actin (Dilution at 
a ratio of 1:1000) for overnight at $4^{\circ} \mathrm{C}$. Later horseradish peroxidase-conjugated secondary antibody was used to incubate for the period of $60 \mathrm{~min}$ at room temperature with total protein. Image J software was used to estimate the band density.

\section{Determination of mediators of inflammation}

ELISA kits were used to determine the concentration of mediators of inflammation such as INF- $\gamma$, IL-1 $\beta$ and TNF- $\alpha$ in the blood of TBI injured rats.

\section{Statistical Analysis}

All data were expressed as mean \pm SEM ( $n$ $=10$ ). The statistical analysis was performed using one way ANOVA. Post-hoc comparison of means was carried out by Dunnett's post hoc test (Gradpad prism 6.1., CA, USA) multiple comparisons. The level of statistical significance was set at $\mathrm{P}<0.05$.

\section{Results}

Stachydrine reduces the mNSS and percentage of water content in the brain

Fig.1. Shows stachydrine effect on the neurological function and percentage of water content in the brain of TBI injured rats. mNSS suggested that the score was enhanced in TBI groups than normal group. Moreover percentage of water content in the brain was also increased in TBI group than normal group. However treatment with stachydrine ameliorates the altered mNSS and percentage of water content in the brain of TBI rats.

\section{Stachydrine ameliorates the} cognitive dysfunction

Assessment of memory impairment in stachydrine treated group in TBI injured rats was shown in Fig.2. Percentage number of crossing and time spent in the target quadrant was found to be less and escape latency was more in TBI group than normal group. Off note, stachydrine reported to improve the memory as percentage number of crossing in TBI injured rats. Time spent in the target quadrant was enhanced and escape latency was reduced in stachydrine group than normal group.
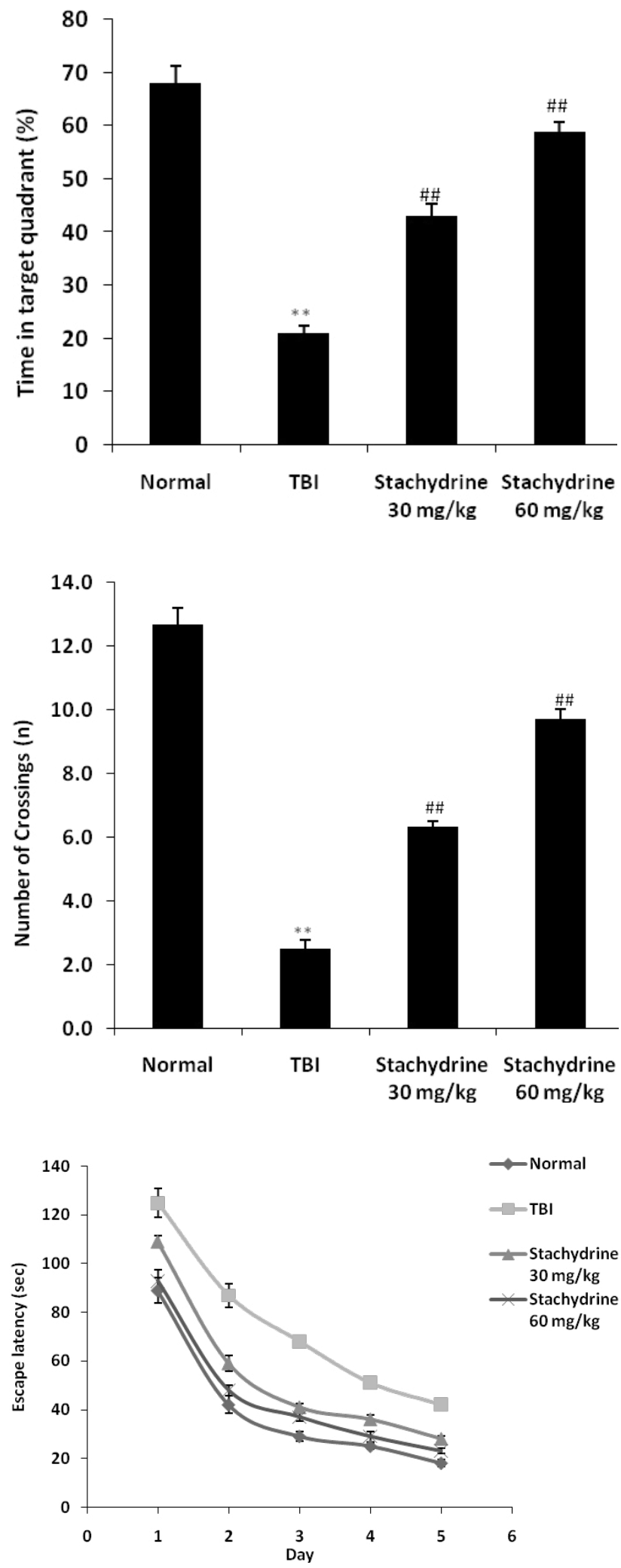

Fig. 2. Stachydrine ameliorate the cognitive dysfunction in TBI injured rats by MWM Mean \pm SEM $(n=10)$, ** $\mathrm{p}<0.01$ than normal group; ${ }^{\# \#} \mathrm{p}<0.01$ than TBI group 


\section{Stachydrine ameliorate the neuronal apoptosis}

Nissl staining was done on the cortical tissues to determine the number of apoptotic neurons (Fig.3.). It was seen that the percentage of apoptotice neurons was found more in TBI group (81.6\%) than normal group (5.3\%). However stachydrine treatment reduces the percentage of apoptotice neurons upto $32.4 \%$ in TBI injured rats.

\section{Stachydrine effect on PI3K/ mTOR/ Akt signaling pathway}

Rats treated with stachydrine assessed for the expressions of $\mathrm{PI} 3 \mathrm{~K} / \mathrm{mTOR} / \mathrm{Akt}$ proteins by western blot assay (Fig.4.). Expression of $\mathrm{p}$-mTOR/t-mTOR, p-Akt/t-Akt and p-PI3K/t-PI3K was decreased significantly $(p<0.01)$ in the brain tissues of TBI group than normal group. There was reduction in the expression of $\mathrm{p}-\mathrm{mTOR} / \mathrm{t}-\mathrm{mTOR}$, $\mathrm{p}$-Akt/t-Akt and p-PI3K/t-PI3K in brain tissues of stachydrine treated group than TBI group.

\section{Stachydrine effect on LC-3, TLR-4 and NF-kB}

Stachydrine ameliorate the altered expressions of NF-KB, TLR- 4 and LC-3 proteins in the brain tissues of TBI injured rats was shown in Fig. 5. There was enhancement of expression of LC-3, TLR-4 and NF-KB protein in the tissue homogenate in the TBI rats than normal group. However treatment with stachydrine lowers the expression of NF-KB, TLR- 4 and LC-3 proteins in tissue homogenate than TBI group.

\section{Discussion}

TBI progress into secondary injury as several pathogenesis such as inflammatory and apoptotic pathway involved in it [12]. Present study evaluates the protective effect of stachydrine in the treatment of TBI. Effect of stachydrine was determined by estimating mNSS, percentage of water content in the brain and cognitive dysfunction in TBI rats. Moreover western blot assay, histopathology and ELISA tests were used to determine the effect of stachydrine on TBI injured rats.

Report reveals that TBI induces the edema in brain which was estimated by determining the content of water in the brain [13]. Moreover Secondary injury in TBI develops the cognitive dysfunction, memory impairment and enhances the mNSS [14]. Data of the study also reveals that memory impairment and mNSS was enhanced in TBI group which supported by previously published reports. It was observed that stachydrine ameliorates the impaired memory and mNSS in TBI injured rats. Literature reveals that many of the drugs show protective effect against the neuronal injury by modulating the PI3K/m-TOR/Akt pathway [15].

$\mathrm{PI} 3 \mathrm{~K} / \mathrm{m}$-TOR/Akt pathway activation results in the initiates of cell proliferation and decreases the apoptosis [16]. It was reported that in neuronal injury expressions of PI3k/ Akt enzymes reduces, which further controls
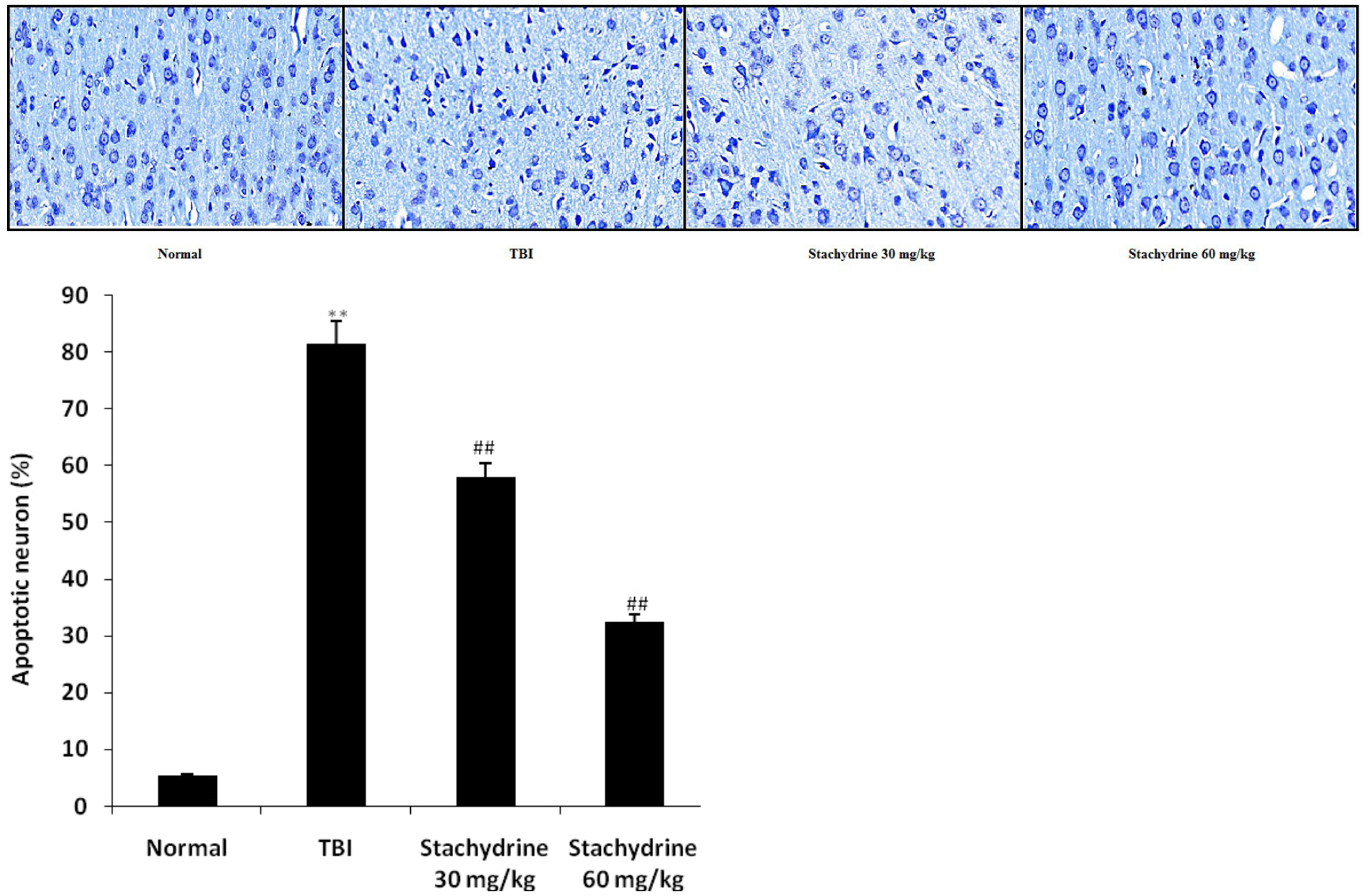

Fig. 3. Stachydrine ameliorate the neuronal apoptosis in the cortical tissues of TBI injured rats by Nissl staining Mean \pm SEM $(n=10),{ }^{* *} p<0.01$ than normal group; ${ }^{\# \# ~} p<$ 0.01 than TBI group 


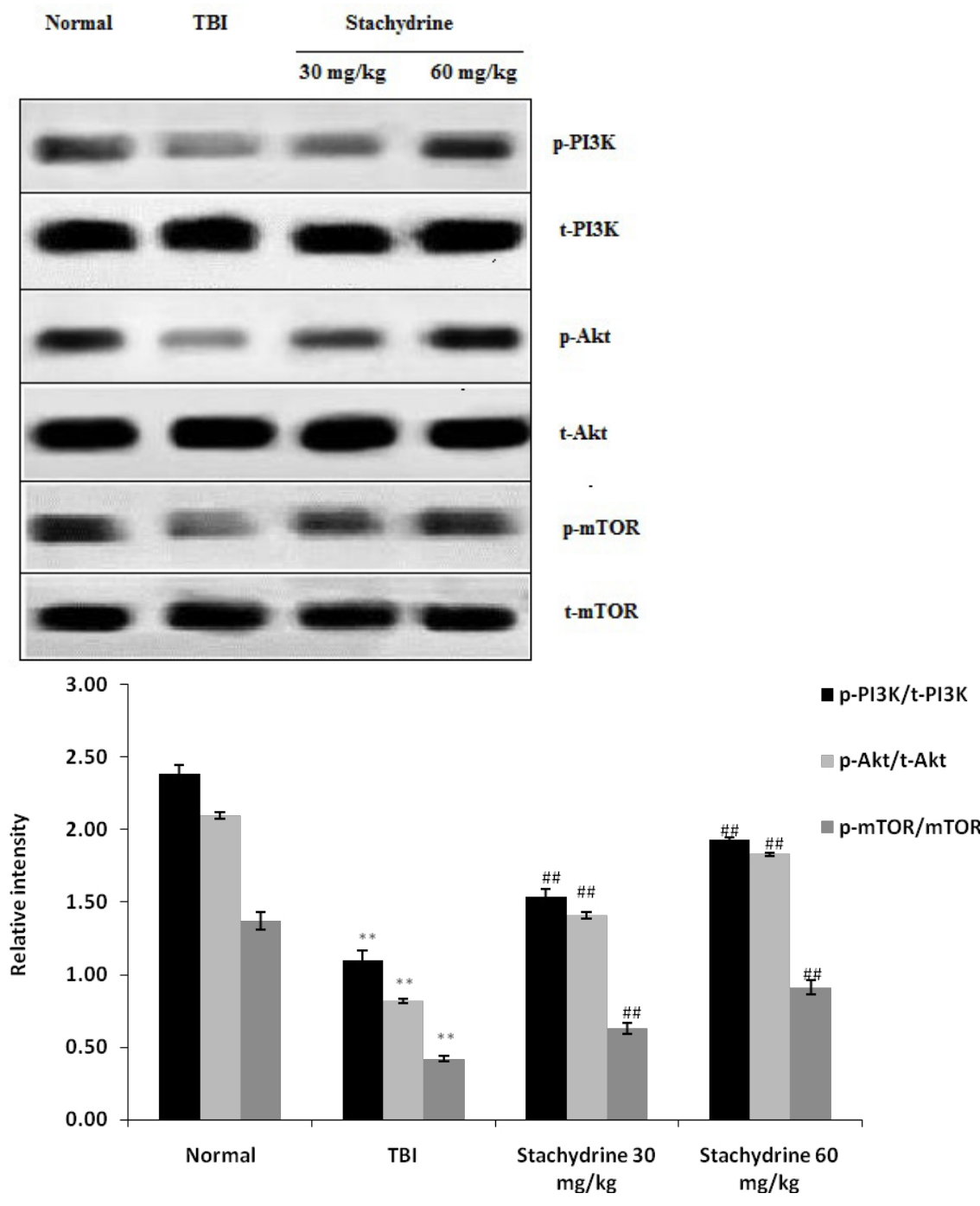

Fig. 4. Stachydrine ameliorate the altered expressions of $\mathrm{PI} 3 \mathrm{~K} / \mathrm{mTOR} /$ Akt proteins in the cortical tissues of TB injured rats Mean \pm SEM $(n=10)$, ${ }^{* *} p<0.01$ than normal group; ${ }^{\# \#} p<0.01$ than TBI group the apoptosis and enhances the proliferation in neuronal cells [17]. Drug used for the management of neuronal injury has role on the modulation of $\mathrm{PI} 3 \mathrm{~K} / \mathrm{m}$-TOR/Akt pathway [18]. Data of our study reveals that stachydrine activates the $\mathrm{PI} 3 \mathrm{~K} / \mathrm{m}$-TOR/Akt pathway by stimulating the expression of $\mathrm{PI} 3 \mathrm{~K} / \mathrm{m}$-TOR/Akt proteins in the brain tissues of TBI injured rats.

Moreover inflammation also has a proven role in the pathogenesis of secondary injury in TBI. Inflammatory mediators such as IL-1 $\beta$ and TNF-a release were enhanced by activating NF$\mathrm{kB}$ signaling pathway due to stimulation of TLR4 receptors [19]. Thereby NF-KB/TLR-4 pathway has shown the role in the development of $\mathrm{TBI}$ [20]. Data of the present investigation reveals that stachydrine ameliorate the altered expression of NF-KB/TLR-4 protein and level of inflammatory mediators in the blood of TBI rats.

\section{Conclusion}

In conclusion, data of this investigation supports that stachydrine protects the neuronal injury in TBI injured rats. It has shown strong anti-inflammatory and anti apoptotic action by ameliorating the altered $\mathrm{PI} 3 \mathrm{~K} / \mathrm{m}$-TOR/ Akt and NF-KB/TLR-4 pathway respectively. Result of this study has shown that stachydrine could be used clinically for the management of neuronal injury.

\section{Acknowledgement}

All authors of this manuscript are thankful to The First Affiliated Hospital of Nanchang University, China for providing the necessary facility for the presented work. 

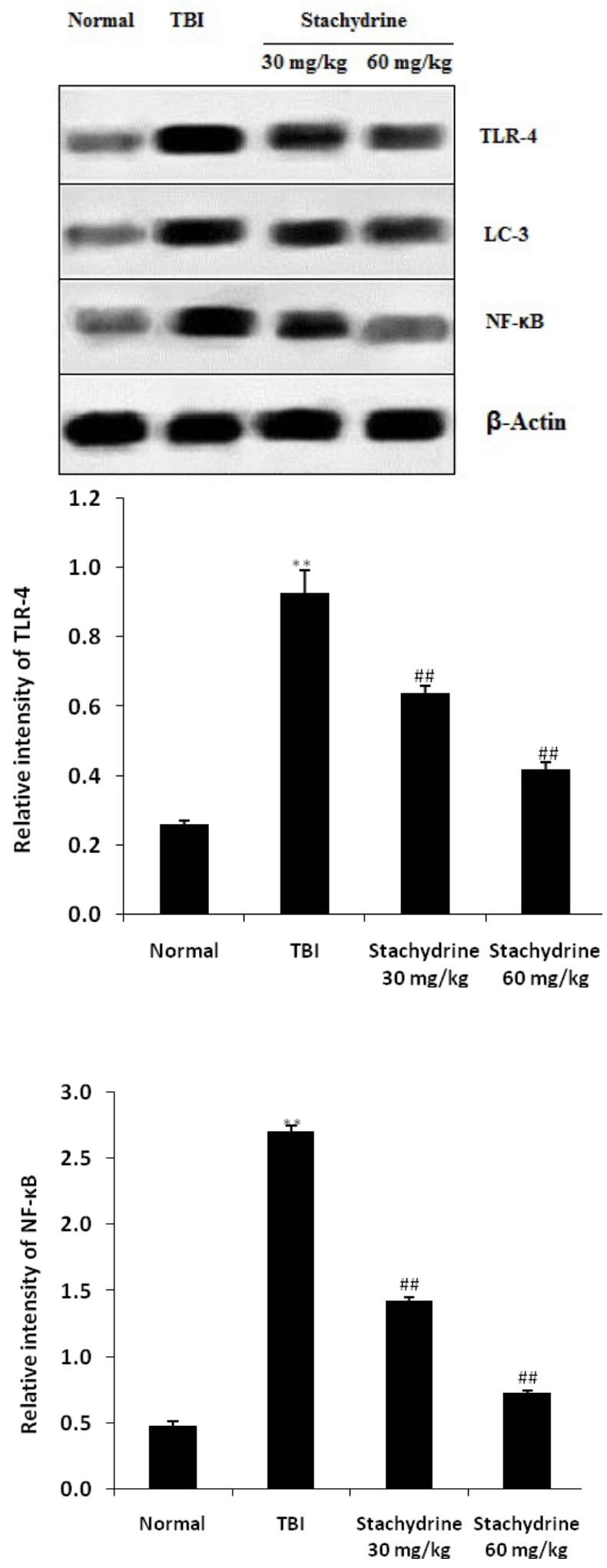

Fig. 5. Stachydrine ameliorate the altered expressions of NF-KB, TLR-4 and LC-3 proteins in the brain tissues of TBI injured rats Mean \pm SEM $(n=10)$, ** $p<0.01$ than normal group; ${ }^{\# \#} \mathrm{p}<0.01$ than TBI group 


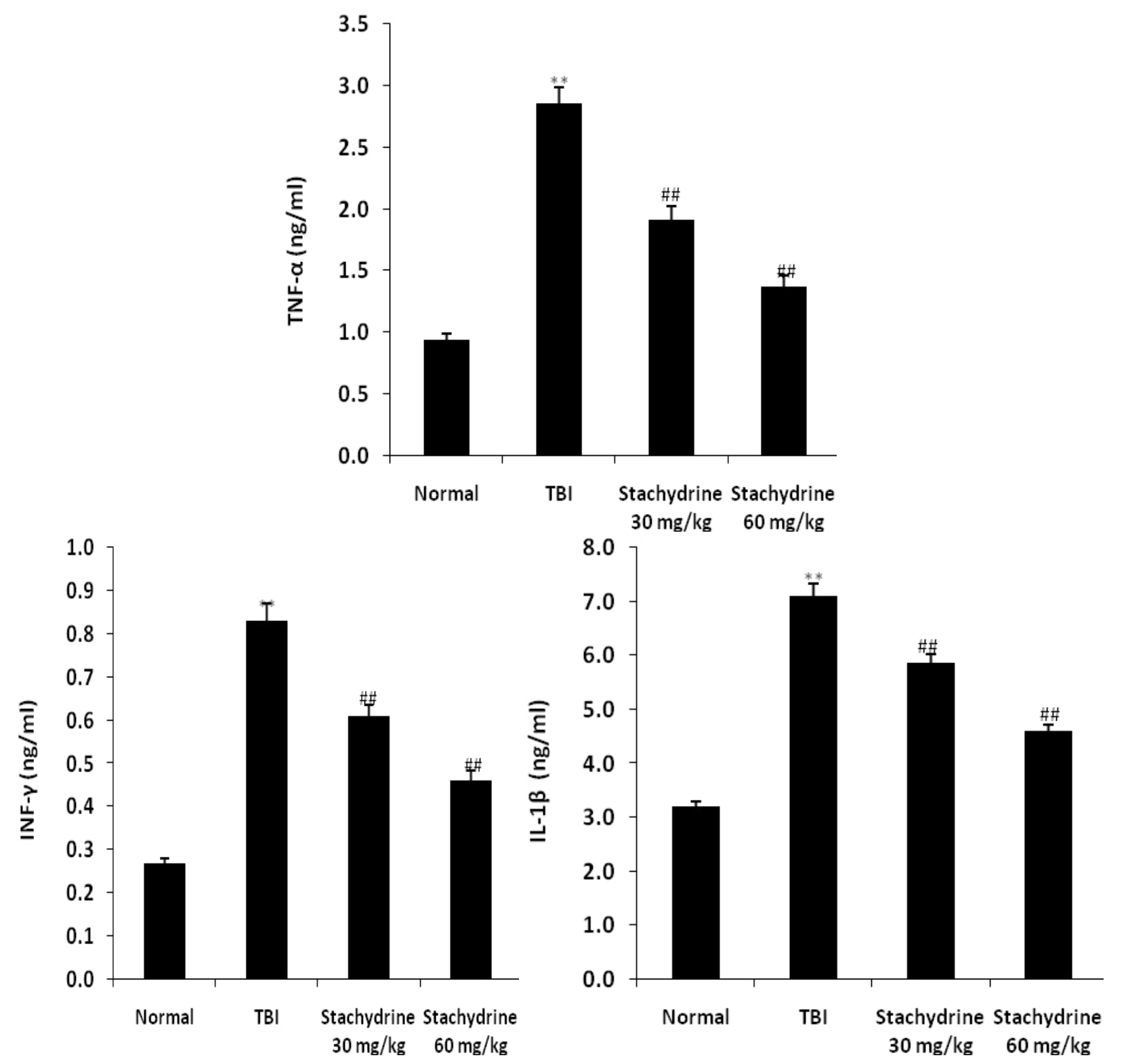

Fig. 6. Stachydrine ameliorate the altered level of inflammatory mediators in the blood of TBI injured rats Mean \pm SEM $(n=10)$, ** $p<0.01$ than normal group; ${ }^{* \#} p<0.01$ than TBI group

References

[1] Popescu C, Anghelescu A, Daia C, Onose G. Actual data on epidemiological evolution and prevention endeavours regarding traumatic brain injury. Journal of Medicine and Life. 2015; 8(3): 272 - 277.

[2] Daneshvar DH, Nowinski CJ, McKee A, Cantu RC. The Epidemiology of Sport-Related Concussion. Clinics in sports medicine. 2011; 30(1): $1-17$.

[3] Mckee AC, Daneshvar DH. The neuropathology of traumatic brain injury. Handbook of clinical neurology. 2015; 127: 45 - 66.

[4] Mikhed Y, Daiber A, Steven S. Mitochondrial Oxidative Stress, Mitochondrial DNA Damage and Their Role in Age-Related Vascular
Dysfunction. Olson L, Ross JM, Coppotelli G, eds. International Journal of Molecular Sciences. 2015; 16(7): 15918 - 15953.

[5] He H, Liu W, Zhou Y, et al. Sevoflurane post-conditioning attenuates traumatic brain injury-induced neuronal apoptosis by promoting autophagy via the PI3K/AKT signaling pathway. Drug Design, Development and Therapy. 2018; 12:629- 638.

[6] Zhang C, Shan X-L, Liao Y-L, et al. Effects of stachydrine on norepinephrine-induced neonatal rat cardiac myocytes hypertrophy and intracellular calcium transients. BMC Complementary and Alternative Medicine. 2014; 14: 474. 
[7] Zhang RH, Liu ZK, Yang DS, Zhang XJ, Sun HD, Xiao WL., Phytochemistry and pharmacology of the genus Leonurus : The herb to benefit the mothers and more, Phytochemistry. 2018; 147: 167 183.

[8] Hu Y, Mao A, Yu Z, He K., Anti-endotoxin and anti-inflammatory effects of Chinese herbal medicinal alkaloid ingredients in vivo. Microb Pathog. 2016; 99: 51 - 55.

[9] Rathee $P$, Rathee $D$, Rathee $D$, Rathee S., In vitro anticancer activity of stachydrine isolated from Capparis decidua on prostate cancer cell lines. Nat Prod Res. 2012; 26(18): 1737 - 40.

[10] Chen $\mathrm{H}-\mathrm{H}$, Zhao P, Zhao W-X, et al. Stachydrine ameliorates pressure overload-induced diastolic heart failure by suppressing myocardial fibrosis. American Journal of Translational Research. 2017; 9(9): 4250 $-4260$.

[11] Zhao L, Wu D, Sang M, Xu Y, Liu Z, Wu Q., Stachydrine ameliorates isoproterenol-induced cardiac hypertrophy and fibrosis by suppressing inflammation and oxidative stress through inhibiting NF$\mathrm{KB}$ and JAK/STAT signaling pathways in rats. Int Immunopharmacol. 2017; 48: 102 - 109.

[12] Xiong Y, Mahmood A, Chopp M. Current understanding of neuroinflammation after traumatic brain injury and cell-based therapeutic opportunities. Chinese Journal of Traumatology. 2018; 21(3): 137 - 151.

[13] Michinaga S, Koyama Y. Pathogenesis of Brain Edema and Investigation into Anti-Edema Drugs. Jia X, ed. International Journal of Molecular Sciences. 2015; 16(5): 9949 - 9975.
[14] Barman A, Chatterjee A, Bhide R. Cognitive Impairment and Rehabilitation Strategies After Traumatic Brain Injury. Indian Journal of Psychological Medicine. 2016; 38(3): 172 - 181.

[15] Li X, Hu X, Wang J, et al. Inhibition of autophagy via activation of $\mathrm{PI} 3 \mathrm{~K} / \mathrm{Akt} / \mathrm{mTOR}$ pathway contributes to the protection of hesperidin against myocardial ischemia/reperfusion injury. International Journal of Molecular Medicine. 2018; 42(4): 1917 - 1924.

[16] Saji M, Ringel MD. The PI3K-AKT-mTOR pathway in initiation and progression of thyroid tumors. Molecular and cellular endocrinology. 2010; 321(1): 20 - 28.

[17] Jellusova J, Rickert RC. The PI3K Pathway in B Cell Metabolism. Critical reviews in biochemistry and molecular biology. 2016; 51(5): 359 378.

[18] Kitagishi Y, Kobayashi M, Kikuta K, Matsuda S. Roles of PI3K/AKT/ GSK3/mTOR Pathway in Cell Signaling of Mental Illnesses. Depression Research and Treatment. 2012; 2012: 752563.

[19] Yao L, Kan EM, Lu J, et al. Toll-like receptor 4 mediates microglial activation and production of inflammatory mediators in neonatal rat brain following hypoxia: role of TLR4 in hypoxic microglia. Journal of Neuroinflammation. 2013; 10: 23.

[20] Chen X, Wu S, Chen C, et al. Omega-3 polyunsaturated fatty acid supplementation attenuates microglial-induced inflammation by inhibiting the HMGB1/TLR4/NF-KB pathway following experimental traumatic brain injury. Journal of Neuroinflammation. 2017; 14: 143. 